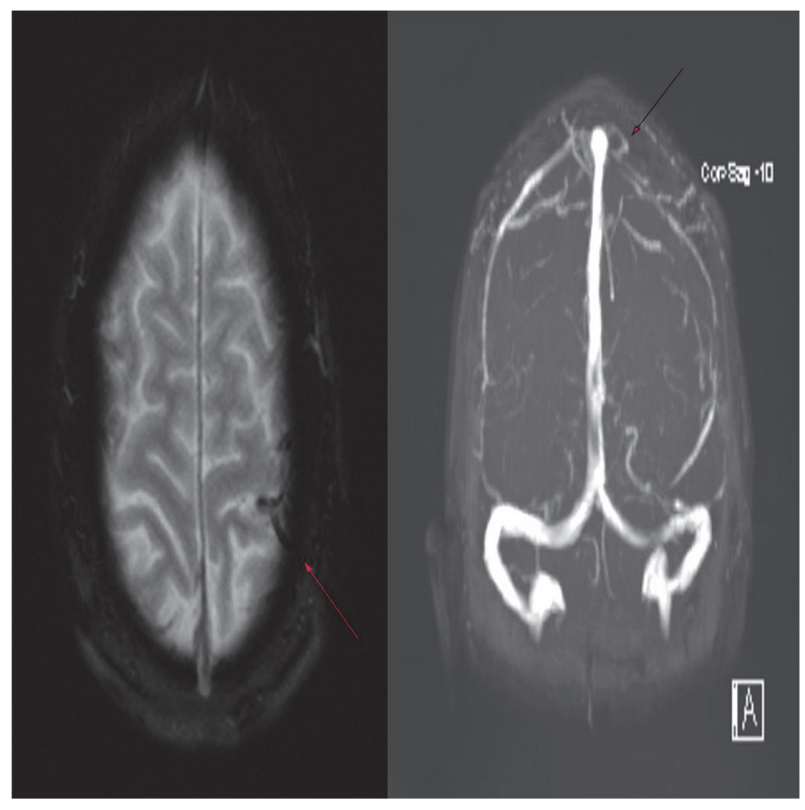

Abstract E-048 Figure 1

Materials A case presentation with Pubmed search of review articles and case reports.

Results The patient was asymptomatic before his first seizure. His seizure semiology consisted of involuntary clonic activity of his right arm followed by generalization and loss of consciousness. He had multiple episodes en route to the hospital lasting less than a minute with full regain of consciousness in between. He did not complain of a headache on the day of admission. CTH was negative, MRI with and without contrast with MRV showed DWI/ADC signal change on the Left frontoparietal region with hypointensity on GRE sequences correlated with absence signal on MRV at the same region (Arrows in Figure). CT abdomen and pelvis with contrast showed findings suggestive of active colitis. Lumbar Puncture opening pressure and CSF profile were within normal limits. Blood count and anemia studies showed platelet counts of $50 \mathrm{~K} / \mathrm{uL}$. Hypercoagulability testing only showed only atypical P-ANCA staining seen in Ulcerative Colitis patients. He was started on levetiracetam, high dose steroids with $1 \mathrm{mg} /$ $\mathrm{kg}$ Enoxaparin every 12 hours. He became seizure free but continued to have progression in right arm weakness with drowsiness and was found to have new bilateral venous infarct in parietal regions on day five post admission. He underwent a diagnostic angiogram that only showed partial resolution of flow in the vein of Trolard. The patient was stable afterward and was discharged to rehab. Literature review estimated the prevalence of CVT in UC patients to be around 6\%, with the most common headache (80\%). The superior sagittal sinus was the most common site of CVT $(50 \%)$. Most patients were treated with anticoagulation regimens ( heparin or low molecular weight heparin) with a good prognosis.

Conclusion Clinicians need to have a low threshold for venous imaging workup in UC patients with acute neurological complaints.

Disclosures A. Kerro: None.

\section{E-049 NORMALIZED INTRAPLAQUE HEMORRHAGE SIGNAL ON MP-RAGE AS A MARKER FOR ACUTE ISCHEMIC NEUROLOGICAL EVENTS}

${ }^{1}$ A Larson*, 'W Brinjikji, 'N Kroll, 'L Savastano, 'J Huston, IJ Benson. 'Radiology, Mayo Clinic, Rochester, MN: ${ }^{2}$ Neurosurgery, Mayo Clinic, Rochester, MN

\subsection{6/neurintsurg-2021-SNIS. 144}

Purpose This study sought to validate whether the signal intensity ratio (SIR) of carotid intraplaque hemorrhage (IPH) was associated with acute ischemic neurologic events.

Methods A retrospective review was completed of consecutive patients that underwent neck MRAs using magnetization prepared rapid gradient echo (MP-RAGE) and T1-CUBE sequences between 2017 and 2020. Patients with MR-evidence of IPH were included. SIRs were measured by comparing the maximum IPH signal with the mean intramuscular signal from the adjacent SCM. Patients were stratified into ischemic or non-ischemic groups based on the presence of acute ipsilateral ischemic events (stroke, retinal artery occlusion). Logistic regression analysis was performed to determine if increasing IPH SIR was associated with an increased risk of ipsilateral ischemic events.

Results Of 85 included patients (85 arteries), 66 were male (77.6\%). Mean age was $71.0(\mathrm{SD} \pm 11.1)$. There were 70 arteries with IPH that were ipsilateral to an ischemic event, and 15 that belonged to a patient without an ischemic event. No association was found between increasing IPH SIR seen on MP-RAGE (OR: 0.82; 95\% CI: 0.58-1.4; P = 0.43) or T1-CUBE sequences (OR: 0.85 ; 95\% CI: 0.53-1.5; P $=0.56$ ). Conclusions There was no association between the SIR of IPH and acute ischemia on either MP-RAGE or T1-CUBE sequences. Further investigation is required prior to widespread acceptance of SIR as a predictive imaging marker of symptomatic carotid plaque.

Disclosures A. Larson: None. W. Brinjikji: None. N. Kroll: None. L. Savastano: None. J. Huston: None. J. Benson: None.

\section{E-050 Y-CONFIGURATION OF NEUROFORM ATLASTM STENTS FOR TREATMENT OF WIDE-NECK INTRA-CRANIAL ANEURYSMS}

${ }^{1} \mathrm{~A}$ Jadhav*${ }^{*}{ }^{2} \mathrm{~S}$ Desai, ${ }^{3} \mathrm{R}$ Hanel, ${ }^{4} \mathrm{D}$ Frei, ${ }^{5} \mathrm{~A}$ Khaldi, ${ }^{6} \mathrm{~S}$ Hetts, ${ }^{7} \mathrm{O}$ Zaidat, ${ }^{8} \mathrm{~B}$ Jankowitz. ${ }^{1}$ University of Pittsburgh Medical Center, Pittsburgh, PA; ${ }^{2}$ Neurology, University of Pittsburgh Medical Center, Pittsburgh, PA; ${ }^{3}$ Neurosurgery, Lyerly Neurosurgery, Jacksonville, FL; ${ }^{4}$ Radiology, Swedish Medical Center, Englewood, CO; ${ }^{5}$ Wellstar Medical Group, Marietta, GA; ${ }^{6}$ Radiology, UCSF Medical Center, San Francisco, CA; ${ }^{7}$ Mercy Health, Toledo, $\mathrm{OH}_{;}{ }^{8}$ Neurosurgery, Cooper University, Camden, NJ

\subsection{6/neurintsurg-2021-SNIS. 145}

Introduction Endovascular management of wide-necked bifurcation aneurysms poses a therapeutic challenge as coiling often requires the use of multiple adjunctive stent constructs to achieve successful embolization without compromising parent vessel integrity. The Neuroform Atlas Stent System is a novel low-profile, intraluminal remodeling device. The study aims to investigate the safety and efficacy of the Y-configuration constructs with the ATLAS stent for aneurysm coil embolization. 